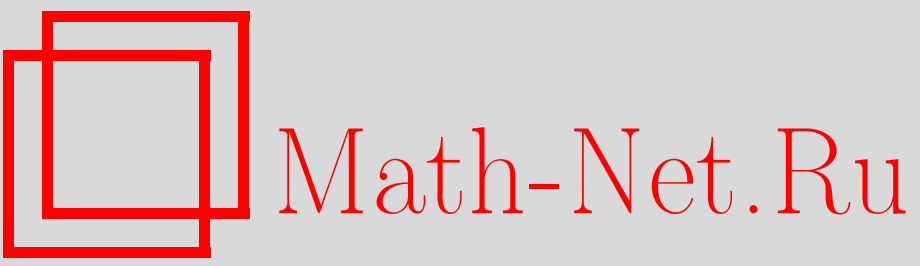

А. М. Ковалева, Бифуркации решений одного уравнения с отклоняющимся пространственным аргументом, Итоги науки и техн. Сер. Соврем. мат. и ее прил. Темат. обз., 2019, том 168, 33-44

DOI: https://doi.org/10.36535/0233-6723-2019-168-33-44

Использование Общероссийского математического портала Math-Net.Ru подразумевает, что вы прочитали и согласны с пользовательским соглашением

http://www.mathnet.ru/rus/agreement

Параметры загрузки:

IP: 3.82 .47 .9

26 апреля 2023 г., 02:32:07 


\title{
БИФУРКАЦИИ РЕШЕНИЙ ОДНОГО УРАВНЕНИЯ С ОТКЛОНЯЮЩИМСЯ ПРОСТРАНСТВЕННЫМ АРГУМЕНТОМ
}

\author{
(c) 2019 г. $\quad$ А. M. КОВАЛЕВА
}

\begin{abstract}
АннотАция. Рассмотрена периодическая краевая задача для одного уравнения с отклоняющимся пространственным аргументом. Данное уравнение описывает фазу световой волны в световых резонаторах с распределенной обратной связью. Оптические системы такого типа используются в компьютерных технологиях и в исследовании лазерных пучков. Поставленная краевая задача рассматривалась при двух значениях пространственного отклонения. В работе рассмотрен ряд бифуркационных задач коразмерностей 1 и 2. Для их решения использовались такие методы исследования динамических систем, как метод нормальных форм Пуанкаре-Дюлака, метод интегральных многообразий и асимптотические формулы. Изучен вопрос об устойчивости ряда однородных состояний равновесий. Получены асимптотические формулы пространственно неоднородных решений и условия их устойчивости.
\end{abstract}

Ключевъе слова: функционально-дифференциальное уравнение, периодическая краевая задача, устойчивость, бифуркация, асимптотика, световой резонатор.

\section{BIFURCATIONS OF SOLUTIONS TO EQUATIONS WITH DEVIATING SPATIAL ARGUMENTS}

\author{
(c) 2019 A. M. KOVALEVA
}

\begin{abstract}
A periodic boundary-value problem for an equation with deviating spatial argument is considered. This equation describes the phase of a light wave in light resonators with distributed feedback. Optical systems of this type are used in computer technologies and in the study of laser beams. The boundary-value problem was considered for two values of spatial deviations. In the work, bifurcation problems of codimensions 1 and 2 were analyzed by various methods of studying dynamical systems, for example, the method of normal Poincaré-Dulac forms, the method of integral manifolds, and asymptotic formulas. The problem on the stability of certain homogeneous equilibrium states is examined. Asymptotic formulas for spatially inhomogeneous solutions and conditions for their stability are obtained.
\end{abstract}

Keywords and phrases: functional-differential equation, periodic boundary-value problem, stability, bifurcation, asymptotics, light resonator.

AMS Subject Classification: 34K18, 34K21, 39A28

1. Введение. В работе рассматривается одномерная по пространству модель, описывающая фазу световой волны в световых резонаторах с распределенной обратной связью. Такие оптические системы используются в компьютерных технологиях и в исследовании лазерных пучков:

$$
u_{t}+u=d u_{x x}+K\left(1+\gamma \cos u_{h}\right) .
$$

Работа выполнена при поддержке Российского фонда фундаментальных исследований (проект № 18-01-00672). 
В данной модели $u(t, x)$ - фаза световой волны, $h$-угол поворота поля на контуре обратной связи, причем $u_{h}=u(t, x+h), K>0$ - коэффициент нелинейности, зависящий от интенсивности входного поля, $d>0$-коэффициент диффузии, $\gamma \in(0,1)$ - видность интенсивности картины.

Дополним задачу периодическим краевым условием

$$
u(t, x)=u(t, x+2 \pi)
$$

и начальным условием

$$
u(0, x)=f(x) .
$$

Пусть $f(x) \in Q_{2}(\delta)$, где $Q_{2}(\delta)$ - достаточно малый шар гильбертова пространства $\mathbb{H}_{2}^{2}$ (пространства Соболева $2 \pi$-периодических функций, имеющих интегрируемые с квадратом обобщенные производные до второго порядка включительно). Такое условие позволяет утверждать, что поставленная смешанная задача (1)-(3) локально корректно разрешима (см. $[7,13])$.

Краевая задача (1), (2) изучалась в $[2,3,10-12]$, но при иных предположениях и в иной постановке. Так, например, в $[2,3,10]$ рассматривался случай, когда коэффициент диффузии мал, что приводит к сингулярно возмущенному уравнению параболического типа. Для изучения такого варианта был использован метод квазинормальных форм. В $[11,12]$ изучался регулярный случай $(d$-величина порядка 1$)$, но в них авторы ограничились изучением некоторых вариантов, при которых реализуются условия бифуркационной теоремы Андронова-Хопфа (см. $[1,4,6,8,9])$. В частности, в указанных работах не изучались бифуркационные задачи коразмерности 2.

2. Линейный анализ. Обозначим через $а$ состояния равновесия краевой задачи (1), (2), т.е. $u=a=$ const. Следовательно, $a$ можно найти как корни уравнения

$$
a=K(1+\gamma \cos a) .
$$

Уравнение (4) при всех допустимых $K$ и $\gamma$ имеет по крайней мере один корень $a>0$, но может иметь и большее их число. При $K=\pi / 2+\pi m, m=0,1,2, \ldots$, оно имеет счетный набор корней

$$
a_{m}=\frac{\pi}{2}+\pi m, \quad m=0,1,2, \ldots
$$

Отметим, что в силу положительности $K$ корни $a_{m}$ тоже могут быть только положительными.

Будем рассматривать два случая для выбора таких корней (состояний равновесия):

$$
\begin{aligned}
& a_{k}=\frac{\pi}{2}+2 \pi k, \\
& a_{p}=\frac{3 \pi}{2}+2 \pi p,
\end{aligned}
$$

где $k, p=0,1,2, \ldots$, т.е. состояния равновесия $a_{m}$ разделим на два типа: $a_{k}$ и $a_{p}$.

Далее будем изучать окрестность таких состояний равновесия и, в частности, вопрос об их устойчивости в смысле нормы пространства начальных условий $\mathbb{H}_{2}^{2}$ для краевой задачи (1), (2).

Положим $u(t, x)=a_{m}+v(t, x), m=0,1,2, \ldots$ В результате получим вспомогательную краевую задачу

$$
\begin{gathered}
v_{t}=d v_{x x}-v+b_{m}\left(v_{h}-\frac{v_{h}^{3}}{3 !}+\frac{v_{h}^{5}}{5 !}+\ldots\right), \quad m=0,1,2, \ldots, \\
v(t, x)=v(t, x+2 \pi),
\end{gathered}
$$

где $v=v(t, x), v_{h}=v(t, x+h), b_{m}=K \gamma \sin a_{m}$, а точками обозначены члены тейлоровского разложения функции $\cos \left(v_{h}+a\right)$, имеющие более высокий порядок малости по сравнению с выписанными $\left(o\left(v_{h}^{3}\right)\right)$. Сразу отметим, что $b_{m}=\beta=K \gamma>0$, если $a_{m}=a_{k}$, и $b_{m}=-\beta$, если $a_{m}=a_{p}$.

Изучим вопрос об устойчивости нулевого решения краевой задачи (5), (6). Для этого рассмотрим ее линеаризованный вариант. В зависимости от выбора состояния равновесия, мы получаем два типа таких задач, которые целесообразно рассмотреть отдельно. 
При выборе состояний равновесия первого типа $\left(a_{k}\right)$ получим следующую краевую задачу:

$$
\begin{gathered}
v_{t}=A_{1}(\beta) v=d v_{x x}-v-\beta v_{h}, \quad \beta=K \gamma>0, \\
v(t, x)=v(t, x+2 \pi) .
\end{gathered}
$$

Во втором случае $\left(a=a_{p}\right)$ получим краевую задачу

$$
\begin{gathered}
v_{t}=A_{2}(\beta) v=d v_{x x}-v+\beta v_{h}, \quad \beta=K \gamma>0, \\
v(t, x)=v(t, x+2 \pi) .
\end{gathered}
$$

Вопрос об устойчивости решений краевых задач $(7),(8)$ и $(9),(10)$ решается после нахождения собственных значений линейных дифференциальных операторов $A_{1}(\beta)$ и $A_{2}(\beta)$. Функции $\exp (\operatorname{in} x), n \in \mathbb{Z}$ являются собственными функциями обоих операторов.

Нетрудно убедиться, что оператор $A_{1}(\beta)$ имеет собственные значения

$$
\lambda_{n, 1}(\beta)=-d n^{2}-1-\beta(\cos n h+i \sin n h),
$$

$\operatorname{Re} \lambda_{n, 1}(\beta)=-d n^{2}-1-\beta \cos n h, \quad \operatorname{Im} \lambda_{n, 1}(\beta)=-\beta \sin n h$.

Во втором варианте, т.е. для $A_{2}(\beta)$, имеем

$$
\begin{gathered}
\lambda_{n, 2}(\beta)=-d n^{2}-1+\beta(\cos n h+i \sin n h), \\
\operatorname{Re} \lambda_{n, 2}(\beta)=-d n^{2}-1+\beta \cos n h, \quad \operatorname{Im} \lambda_{n, 2}(\beta)=\beta \sin n h .
\end{gathered}
$$

Напомним, что решения краевой задачи (7), (8) (соответственно, (9), (10)) асимптотически устойчивы, если $\operatorname{Re} \lambda_{n, 1}(\beta)<0$ (соответственно, $\left.\operatorname{Re} \lambda_{n, 2}(\beta)<0\right), n=0,1,2, \ldots$ При реализации указанного условия нулевое состояние равновесия нелинейной краевой задачи (5), (6) будет асимптотически устойчивым, а если $\operatorname{Re} \lambda_{n_{0}, 1}(\beta)>0\left(\right.$ соответственно, $\left.\operatorname{Re} \lambda_{n_{0}, 2}(\beta)>0\right)$ при некотором $n=n_{0}-$ то неустойчивым. Наконец, пусть $\operatorname{Re} \lambda_{s_{j}, 1}(\beta)=0\left(\right.$ соответственно, $\left.\operatorname{Re} \lambda_{s_{j}, 2}(\beta)=0\right)$ при некоторых $n=s_{1}, s_{2}, \ldots, s_{l}$, а при остальных $n$ все собственные числа оператора лежат в левой комплексной полуплоскости; тогда в задаче об устойчивости нулевого решения нелинейной краевой задачи реализуется критический случай. Далее будем изучать расположение комплексных чисел $\lambda_{n, 1}(\beta)$ и $\lambda_{n, 2}(\beta)$ на комплексной плоскости. Подчеркнем, что собственные значения $\lambda_{n, 1}(\beta), \lambda_{n, 2}(\beta)$ также зависят и от $h$. В работе ограничимся анализом выбранных значений $h$ : $h=\pi / 4, h=\pi / 3$.

Рассмотрим сначала вопрос об устойчивости состояний равновесия второго типа, т.е. рассмотрим вопрос об устойчивости состояния равновесия

$$
a_{p}=\frac{3 \pi}{2}+2 \pi p, \quad p=0,1,2, \ldots
$$

Пусть сначала $h=\pi / 4$. Нетрудно заметить, что $\operatorname{Re} \lambda_{n+8 l, 2}(\beta)<\operatorname{Re} \lambda_{k, 2}(\beta)$, если $n=k+8 m$, $k, m=1,2, \ldots$ Поэтому в данном случае достаточно проанализировать знак действительной части первых восьми собственных значений оператора $A_{2}(\beta)$. Очевидно,

$$
\begin{array}{lll}
\operatorname{Re} \lambda_{0,2}(\beta)=-1+\beta, & \operatorname{Re} \lambda_{1,2}(\beta)=-d-1+\frac{\sqrt{2}}{2} \beta, & \operatorname{Re} \lambda_{2,2}(\beta)=-4 d-1, \\
\operatorname{Re} \lambda_{3,2}(\beta)=-9 d-1-\frac{\sqrt{2}}{2} \beta, & \operatorname{Re} \lambda_{4,2}(\beta)=-16 d-1-\beta, & \operatorname{Re} \lambda_{5,2}(\beta)=-25 d-1-\frac{\sqrt{2}}{2} \beta, \\
\operatorname{Re} \lambda_{6,2}(\beta)=-36 d-1, & \operatorname{Re} \lambda_{7,2}(\beta)=-49 d-1+\frac{\sqrt{2}}{2} \beta . &
\end{array}
$$

Сравнения этих действительных чисел показало, что при $\beta \in(0,1)$ все они отрицательны, а при $\beta>1$ по крайней мере одно из них $\left(\operatorname{Re} \lambda_{0,2}(\beta)\right)$ положительно. Наконец, при $\beta=\beta_{\text {кр }}=1$ и ему соответствует собственная функция $e_{0}=1$.

Аналогичная ситуация для оператора $A_{2}(\beta)$ реализуется при $h=\pi / 3$. Итак, справедливо следующее утверждение. 
Теорема 1. Пусть $h=\pi / 4$ или $h=\pi / 3$. Любое состояние равновесия $a_{p}=3 \pi / 2+2 \pi p$, $p=0,1,2, \ldots$, краевой задачи (1), (2) асимптотически устойчиво, если $\beta \in[0,1)$, и неустойчиво при $\beta \in(1, \infty)$.

Рассмотрим теперь вопрос об устойчивости состояний равновесия

$$
a_{k}=\frac{\pi}{2}+2 \pi k, \quad k=0,1,2, \ldots,
$$

и, следовательно, изучим вопрос о расположении собственных значений оператора $A_{1}(\beta)$.

Пусть сначала $h=\pi / 4$. Как и ранее, просто проверяется справедливость неравенства $\operatorname{Re} \lambda_{n+8 l, 1}(\beta)<\operatorname{Re} \lambda_{n, 1}(\beta)$, если $l=1,2, \ldots$. Поэтому интерес представляют лишь следующие числа:

$$
\begin{array}{lll}
\operatorname{Re} \lambda_{0,1}(\beta)=-1-\beta, & \operatorname{Re} \lambda_{1,1}(\beta)=-d-1-\frac{\sqrt{2}}{2} \beta, & \operatorname{Re} \lambda_{2,1}(\beta)=-4 d-1, \\
\operatorname{Re} \lambda_{3,1}(\beta)=-9 d-1+\frac{\sqrt{2}}{2} \beta, & \operatorname{Re} \lambda_{4,1}(\beta)=-16 d-1+\beta, & \operatorname{Re} \lambda_{5,1}(\beta)=-25 d-1+\frac{\sqrt{2}}{2} \beta, \\
\operatorname{Re} \lambda_{6,1}(\beta)=-36 d-1, & \operatorname{Re} \lambda_{7,1}(\beta)=-49 d-1-\frac{\sqrt{2}}{2} \beta . &
\end{array}
$$

Очевидно, что $\operatorname{Re} \lambda_{k, 1}<0$ при $k=0,1,2,6,7$ для всех значений параметров $\beta, d>0$, а также, что $\operatorname{Re} \lambda_{5,1}(\beta)<\operatorname{Re} \lambda_{3,1}(\beta)$ для всех рассматриваемых $\beta$. Поэтому смена знака при наименьшем из возможных значений параметра $\beta$ возможна лишь у $\operatorname{Re} \lambda_{k, 1}$ при $k=3,4$.

Найдем наименьшее $\beta$, при котором $\operatorname{Re} \lambda_{3,1}, \operatorname{Re} \lambda_{4,1}$ могут обращаться в нуль:

$$
\beta_{\text {min }}=\min \{\sqrt{2}(9 d+1), 16 d+1\},
$$

где $d>0$. Следовательно, анализу подлежат неравенства

$$
16 d+1<\sqrt{2}(9 d+1), \quad 16 d+1>\sqrt{2}(9 d+1) .
$$

Первое из них выполнено, если $d<(2+7 \sqrt{2}) / 94$, а второе, напротив, если $d>(2+7 \sqrt{2}) / 94$.

В первом случае смена устойчивости возможна при выборе собственных значений $\lambda_{4,1}$, а в следующем - собственных значений $\lambda_{3,1}$. Из этих построений вытекает справедливость следующего утверждения.

Теорема 2. Пусть $h=\pi / 4, \beta>0$. Любые состояние равновесия

$$
a_{k}=\frac{\pi}{2}+2 \pi k, \quad k=0,1,2, \ldots,
$$

краевой задачи (1), (2) асимптотически устойчивы, если

$$
\beta<\beta_{*}=\min \{\sqrt{2}(9 d+1), 16 d+1\}, \quad d>0,
$$

или в иной форме

$$
\gamma<\gamma_{\mathrm{kp}}=\frac{\beta_{*}}{\pi / 2+2 \pi k} .
$$

Эти состояния равновесия неустойчивы, если $\beta>\beta_{*}\left(\gamma>\gamma_{\mathrm{kp}}\right)$.

$\mathrm{K}$ формулировке результата уместно добавить, что при $\beta=\beta_{*}\left(\gamma=\gamma_{\text {кр }}\right)$ реализуется критический случай в задаче об устойчивости соответствующего состояния равновесия. Если $d<(2+7 \sqrt{2}) / 94$, то оператор $A_{1}(\beta)$ имеет двукратное нулевое собственное значение, которому отвечают два собственных элемента $\exp ( \pm 4 i x)$. Если же $d>(2+7 \sqrt{2}) / 94$, то оператор $A_{1}(\beta)$ имеет два чисто мнимых собственных значения $\pm i \sigma, \sigma=\beta \sqrt{2} / 2$. Этим собственным значениям отвечают собственные функции $\exp ( \pm 3 i x)$. В данном случае потеря устойчивости происходит колебательным образом и возможна бифуркация цикла.

Наконец, при $d=d_{*}=(2+7 \sqrt{2}) / 94$ и $\beta=\beta_{*}=7(9+8 \sqrt{2}) / 47$ реализуется критический случай коразмерности 2 , когда на мнимой оси находятся пара $\pm i(7(16+9 \sqrt{2})) / 94$ и нулевое собственное число кратности 2. Им соответствуют собственные функции $\exp ( \pm 3 i x)$ и $\exp ( \pm 4 i x)$.

Теперь рассмотрим тот же вопрос при $h=\pi / 3$. Справедливо аналогичное утверждение. 
Теорема 3. Любое состояние равновесия

$$
a_{k}=\frac{\pi}{2}+2 \pi k, \quad k=0,1,2, \ldots,
$$

краевой задачи (1), (2) в случае $h=\pi / 3$ асимптотически устойчиво, если

$$
\beta<\beta_{*}=\min \{8 d+2,9 d+1\},
$$

или в иной форме

$$
\gamma<\gamma_{\mathrm{кр}}=\frac{\beta_{*}}{\pi / 2+2 \pi k} .
$$

Эти состояния равновесия неустойчивы, если $\beta>\beta_{*}\left(\gamma>\gamma_{\mathrm{kp}}\right)$.

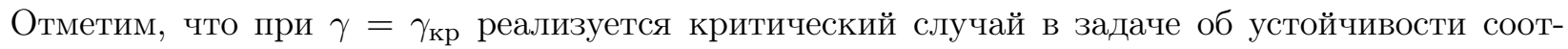
ветствующего состояния равновесия. При $d<1$ реализуется критический случай двукратного нулевого собственного значения с собственными функциями $\exp ( \pm 3 i x)$. При $d>1$ имеет место критический случай пары чисто мнимых собственных значений $\pm i \beta \sqrt{3} / 2$, соответствующих собственным функциям $\exp ( \pm 2 i x)$. Наконец, при $d=1, \beta=10$ реализуется критический случай коразмерности 2 , когда спектру оператора $A_{1}(\beta)$ принадлежат двукратный нуль с собственными функциями $\exp ( \pm 3 i x)$ и пара чисто мнимых собственных значений $\pm 5 i \sqrt{3}$, которые отвечают собственной функции $\exp ( \pm 2 i x)$.

3. Бифуркационная задача в случае пары чисто мнимых корней, близком к критическому. Такой критический случай, когда на мнимой оси может появиться пара простых чисто мнимых собственных значений, реализуется только для состояний равновесия первого типа, т.е. при рассмотрении состояний равновесия $a_{k}=\pi / 2+2 \pi k, k=0,1,2, \ldots$ Напомним, что исследуемый критический случай при $h=\pi / 4$ возникает на третьей моде $(n=3)$, т.е. собственные функции оператора $A_{1}(\beta)$ равны $\exp ( \pm 3 i x)$, а при $h=\pi / 3$ - на второй моде (собственные функции $\exp ( \pm 2 i x))$.

Рассмотрим задачу (5), (6) при $\gamma=\gamma_{\mathrm{kp}}(1-\varkappa \varepsilon), \varkappa= \pm 1, \varepsilon \in\left(\varepsilon, \varepsilon_{0}\right), \varepsilon_{0} \ll 1, s=\varepsilon t$ :

$$
\begin{gathered}
v_{t}=d v_{x x}-v-a_{k} \gamma_{\mathrm{кр}}(1-\varkappa \varepsilon) \sin a_{k}\left(v_{h}-\frac{v_{h}^{3}}{3 !}+\frac{v_{h}^{5}}{5 !}+\ldots\right), \\
v(t, x)=v(t, x+2 \pi) .
\end{gathered}
$$

Положим

$$
A(\varepsilon)=A\left(\gamma_{\mathrm{kp}}(1-\varkappa \varepsilon)\right)=d v_{x x}-v-K \gamma_{\mathrm{kp}}(1-\varkappa \varepsilon) \sin a_{k} v_{h} .
$$

Этот оператор имеет пару собственных значений $\lambda(\varepsilon)=\tau(\varepsilon) \pm i \sigma(\varepsilon)$ :

$$
\tau(\varepsilon)=-d n^{2}-1-K \gamma_{\mathrm{Kp}}(1-\varkappa \varepsilon) \sin a_{k} \cos n h, \quad \sigma(\varepsilon)=-K \gamma_{\mathrm{Kp}}(1-\varkappa \varepsilon) \sin a_{k} \sin n h .
$$

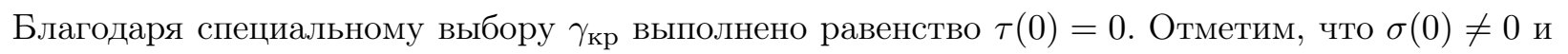

$$
\left.\frac{d \operatorname{Re} \lambda(\varepsilon)}{d \varepsilon}\right|_{\varepsilon=0}=\left.\frac{d \tau(\varepsilon)}{d \varepsilon}\right|_{\varepsilon=0}=K \gamma_{\mathrm{\kappa p}} \varkappa \sin a_{k} \cos n h \neq 0
$$

В результате несложно проверить (см. $[1,9]$ ), что реализуются условия применимости теоремы Андронова-Хопфа. Следовательно, анализ структуры окрестности нулевого состояния равновесия может быть сведен к анализу нормальной формы Пуанкаре--юлака (см. $[8,9])$.

Будем искать решения краевой задачи (11), (12), которые могут быть представлены в следующем виде:

$$
v(t, x, \varepsilon)=\varepsilon^{1 / 2} v_{1}(t, x, s)+\varepsilon v_{2}(t, x, s)+\varepsilon^{3 / 2} v_{3}(t, x, s)+o\left(\varepsilon^{3 / 2}\right) ;
$$

здесь $v_{j}(t, x, s), j=1,2, \ldots$, - достаточно гладкие функции, для которых выполнены следующие свойства:

(i) $v_{j}(t, x+2 \pi, s)=v_{j}(t, x, s), j=1,2, \ldots$;

(ii) $v_{j}\left(t+2 \pi / \sigma_{n}, x, s\right)=v_{j}(t, x, s), j=1,2, \ldots, \sigma_{n}=\operatorname{Im}\left(\lambda_{n, 1}\right)$; 
(iii) для функций $v_{k}(t, x, s)$ при $k=2,3, \ldots$ справедливы равенства

$$
\frac{\sigma_{n}}{(2 \pi)^{2}} \int_{0}^{2 \pi / \sigma_{n}} \int_{0}^{2 \pi} v_{k}(t, x, s) d x d t=\frac{\sigma_{n}}{(2 \pi)^{2}} \int_{0}^{2 \pi / \sigma_{n}} \int_{0}^{2 \pi} v_{k}(t, x, s) \exp \left( \pm i n x \pm i \sigma_{n} t\right) d x d t=0 .
$$

Следуя алгоритму построения нормальной формы, подставим сумму (13) в нелинейное уравнение (11). Приравнивая коэффициенты при $\varepsilon^{1 / 2}$ и $\varepsilon$, получаем две краевые задачи:

$$
\begin{gathered}
v_{1 t}=B v_{1}=d v_{1 x x}-v_{1}-a_{k} \sin a_{k} \gamma_{\mathrm{kp}} v_{h_{1}}, \quad v_{1}(t, x+2 \pi)=v_{1}(t, x) ; \\
v_{2 t}=B v_{2}, \quad v_{2}(t, x+2 \pi)=v_{2}(t, x) .
\end{gathered}
$$

Положим

$$
v_{1}(t, x, s)=z(s) \exp \left(i n x+i \sigma_{n} t\right)+\overline{z(s)} \exp \left(-i n x-i \sigma_{n} t\right) ; \quad \sigma_{n}=\operatorname{Im}\left(\lambda_{n, 1}\right) .
$$

Краевая задача для $v_{2}(t, x, s)$ имеет нулевое решение. Следовательно, для определения $v_{3}$ получим краевую задачу

$$
\begin{aligned}
& v_{3 t}=B v_{3}-v_{1 s}+a_{k} \sin a_{k} \gamma_{\mathrm{kp}}\left[\varkappa v_{h_{1}}+\frac{1}{6} v_{h_{1}}^{3}\right]=B v_{3}+F(t, x, s), \\
& v_{3}(t, x+2 \pi)=v_{3}(t, x) .
\end{aligned}
$$

Для построения нормальной формы воспользуемся условием разрешимости в классе периодических функций применительно к неоднородной краевой задаче (14):

$$
\int_{0}^{2 \pi / \sigma_{n}} \int_{0}^{2 \pi} F(t, x, s) \exp \left( \pm i n x \pm i \sigma_{n} t\right) d x d t=0 .
$$

Применяя это условие к краевой задаче (14), получаем систему, которая является нормальной формой:

$$
z^{\prime}=a_{k} \sin a_{k} \gamma_{\mathrm{\kappa p}} \varkappa \exp (i n h) z+\frac{1}{2} \sin a_{k} \gamma_{\mathrm{\kappa p}} \exp (i n h)|z|^{2} z
$$

Преобразуем нормальную форму (15), положив $z(s)=\rho(s) \exp (i \varphi(s)), \rho(s)>0$. Введем обозначение $g=a_{k} \sin a_{k} \gamma_{\text {кр }} \cos n h$. После несложных вычислений нормальная форма принимает следующий вид:

$$
\left\{\begin{array}{l}
\rho^{\prime}=g\left(\varkappa \rho+\frac{1}{2} \rho^{3}\right), \\
\varphi^{\prime}=a_{k} \sin a_{k} \gamma_{\mathrm{kp}} \sin n h\left(\varkappa+\frac{1}{2} \rho^{2}\right) .
\end{array}\right.
$$

Особую роль при анализе полученной нормальной формы (16) играет уравнение для амплитуды $\rho$. Оно имеет состояние равновесия $S_{0}: \rho_{0}=\sqrt{-2 \varkappa}$, существующее при $\varkappa<0$. Ему соответствует состояние равновесия системы (16): $\rho(s)=\sqrt{-2 \varkappa}, \varphi(s)=\varphi_{0}$, где $\varphi_{0}$ - произвольная действительная постоянная. Стандартным образом определяется, что $S_{0}$ асимптотически устойчиво, когда $g<0$, и неустойчиво при $g>0$.

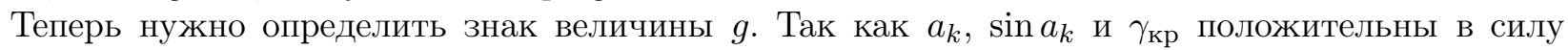
положительности $K$ и выбора $a_{k}$, то $\operatorname{sign} g=\operatorname{sign} \cos n h, \operatorname{ar} \cos n h(\cos 2 \pi / 3$ и $\cos 3 \pi / 4)$ отрицателен, и, следовательно, $g<0$.

Теперь сформулируем полученный результат.

Теорема 4. Состояние равновесия $S_{0}$ дифференциального уравнения для $\rho$ в (16) существует и асимптотически устойчиво при $\varkappa<0$.

Анализ нормальной формы (см. теорему 4) и результаты работ [4-6] приводят к следующему утверждению. 
Теорема 5. Существует такое $\varepsilon_{0}>0$, что при всех $\varepsilon \in\left(0, \varepsilon_{0}\right)$ рассмотренная краевая задача (5), (6) имеет решение с асимптотикой

$$
v(t, x, \varepsilon)=\varepsilon^{1 / 2} \sqrt{-2 \varkappa}(q+\bar{q})+o(\varepsilon), \quad q=\exp \left(i \sigma_{n} t+i n x+i \varphi_{0}\right), \quad \sigma_{n}=\operatorname{Im} \lambda_{n, 1}, \quad \varphi_{0} \in \mathbb{R} .
$$

Это решение существует и асимптотически устойчиво, если $<<0$.

Следствие 1. Асимптотическая формула из теоремь 5 при $h=\pi / 4$ приобретает следующй вид:

$$
v(t, x, \varepsilon)=\varepsilon^{1 / 2} \sqrt{2}\left(\exp \left(3 i x-i(9 d+1) t+i \varphi_{0}\right)+\exp \left(-3 i x+i(9 d+1) t-i \varphi_{0}\right)\right)+o(\varepsilon),
$$

а при $h=\pi / 3-$ вид

$$
v(t, x, \varepsilon)=\varepsilon^{1 / 2} \sqrt{2}\left(\exp \left(2 i x-i \sqrt{3}(4 d+1) t+i \varphi_{0}\right)+\exp \left(-2 i x+i \sqrt{3}(4 d+1) t-i \varphi_{0}\right)\right)+o(\varepsilon) .
$$

4. Критический случай двукратного нуля. Пусть реализуется критический случай коразмерности 1, когда на мнимой оси появляется пара нулевых собственных значений (см. леммы 2 и 3). Описанная ситуация, как и предыдущая, возникает только при рассмотрении оператора $A_{1}(\beta)$. Данный критический случай возникает при $n=4$, если $h=\pi / 4$, и при $n=3$ для $h=\pi / 3$.

В данной ситуации анализ бифуркационной задачи почти повторяет предыдущий раздел. В результате исследования рассматриваемого критического случая, можно сформулировать результат, схожий с теоремой 5. Отличие состоит в том, что этом разделе $\sigma_{n}=0$ для всех $n$.

Приведем асимптотические формулы решений, полученных при анализе данной бифуркационной задачи.

Теорема 6. Асимптотическая формула для пространственно неоднородного состояния равновесия при $h=\pi / 4$ имеет вид

$$
v(t, x, \varepsilon)=\varepsilon^{1 / 2} \sqrt{2}\left(\exp \left(4 i x+i \varphi_{0}\right)+\exp \left(-4 i x-i \varphi_{0}\right)\right)+o(\varepsilon), \quad \varphi_{0} \in \mathbb{R}
$$

a при $h=\pi / 3-$ вид

$$
v(t, x, \varepsilon)=\varepsilon^{1 / 2} \sqrt{2}\left(\exp \left(3 i x+i \varphi_{0}\right)+\exp \left(-3 i x-i \varphi_{0}\right)\right)+o(\varepsilon), \quad \varphi_{0} \in \mathbb{R} .
$$

В каждом из отмеченных случаев краевая задача (5), (6) имеет однопараметрическое семейство пространственных состояний равновесия.

5. Критический случай коразмерности 2. Из линейного анализа вытекает, что такой критический случай реализуется только при рассмотрении состояний равновесия $a_{k}=\pi / 2+2 \pi m$, $k=0,1,2, \ldots$

Если $h=\pi / 4$, то оператор $A_{1}(\beta)$ при

$$
\beta_{\mathrm{\kappa p}}=\frac{7(9+8 \sqrt{2})}{47}, \quad d_{\mathrm{\kappa p}}=\frac{2+7 \sqrt{2}}{94}
$$

имеет пару чисто мнимых собственных значений и нуль кратности 2. Соответствующие им собственные функции имеют вид $\exp ( \pm 3 i x), \exp ( \pm 4 i x)$.

При $h=\pi / 3$, когда $d_{\text {кр }}=1, \beta_{\text {кр }}=10$, реализуется аналогичная ситуация, но на второй и третьей модах. В обоих случаях можно считать выбранной и соответствующую величину $\gamma_{\text {кр }}$

Положим $d=d_{\text {кр }}\left(1-\varkappa_{1} \varepsilon\right)$ и $\gamma=\gamma_{\text {кр }}\left(1-\varkappa_{2} \varepsilon\right)$, где $\varkappa_{1}, \varkappa_{2} \in \mathbb{R}$ произвольны, $\varepsilon \in\left(\varepsilon, \varepsilon_{0}\right), \varepsilon_{0} \ll 1$, $s=\varepsilon t$. Соответствующие моды обозначим ниже. В первом случае $(h=\pi / 4)$ имеем $m=3$, а во втором $(h=\pi / 3)-m=2$. Также отметим, что для обоих пространственных отклонений имеет место равенство $\operatorname{Im} \lambda_{m+1,1}=\sigma_{m+1}=0$ :

$$
\begin{gathered}
v_{t}=d_{\mathrm{\kappa p}}\left(1-\varkappa_{1} \varepsilon\right) v_{x x}-v-a_{k} \gamma_{\mathrm{\kappa p}}\left(1-\varkappa_{2} \varepsilon\right) \sin a_{k}\left(v_{h}-\frac{v_{h}^{3}}{3 !}+\frac{v_{h}^{5}}{5 !}+\ldots\right), \\
v(t, x)=v(t, x+2 \pi) .
\end{gathered}
$$


Найдем решения краевой задачи $(17),(18)$, которые могут быть представлены в следующем виде:

$$
v(t, x, \varepsilon)=\varepsilon^{1 / 2} v_{1}(t, x, s)+\varepsilon v_{2}(t, x, s)+\varepsilon^{3 / 2} v_{3}(t, x, s)+o\left(\varepsilon^{3 / 2}\right) .
$$

Как и в случае коразмерности $1, v_{j}(t, x, s), j=1,2, \ldots$, - достаточно гладкие функции, для которых выполнен ряд свойств:

(i) $v_{j}(t, x+2 \pi, s)=v_{j}(t, x, s), j=1,2, \ldots$;

(ii) функции $v_{j}(t, x, s), j=1,2, \ldots$, являются периодическими по переменной $t$ с периодом $2 \pi / \sigma_{m}$, где $\sigma_{m}=\operatorname{Im} \lambda_{m, 1}$

(iii) для функций $v_{k}(t, x, s), r=2,3, \ldots$, справедливы равенства

$$
\begin{gathered}
\frac{\sigma_{m}}{(2 \pi)^{2}} \int_{0}^{2 \pi / \sigma_{m}} \int_{0}^{2 \pi} v_{k}(t, x, s) d x d t=\frac{\sigma_{m}}{(2 \pi)^{2}} \int_{0}^{2 \pi / \sigma_{m}} \int_{0}^{2 \pi} v_{k}(t, x, s) \exp \left( \pm i m x \pm i \sigma_{m} t\right) d x d t=0, \\
\frac{\sigma_{m}}{(2 \pi)^{2}} \int_{0}^{2 \pi / \sigma_{m}} \int_{0}^{2 \pi} v_{k}(t, x, s) \exp ( \pm i(m+1) x) d x d t=0 .
\end{gathered}
$$

Следуя алгоритму построения нормальной формы, подставим представление (19) в нелинейное уравнение (17). Приравнивая коэффициенты при $\varepsilon^{1 / 2}$, имеем

$$
v_{1 t}=B v_{1}=d_{\mathrm{\kappa p}} v_{1 x x}-v_{1}-a_{k} \sin a_{k} \gamma_{\mathrm{\kappa p}} v_{h_{1}}, \quad v_{1}(t, x+2 \pi, s)=v_{1}(t, x, s) .
$$

Несложно проверить, что функции вида

$$
\begin{aligned}
v_{1}(t, x, s)=z_{1}(s) \exp (i m x+ & \left.i \sigma_{m} t\right)+\overline{z_{1}(s)} \exp \left(-i m x-i \sigma_{m} t\right)+ \\
& +z_{2}(s) \exp (i(m+1) x)+\overline{z_{2}(s)} \exp (-i(m+1) x) ; \quad \sigma_{m}=\operatorname{Im} \lambda_{m, 1},
\end{aligned}
$$

являются решением краевой задачи (20).

Как и в предыдущем случае, $v_{2}=0$. При $\varepsilon^{1 / 2}$ получаем следующую краевую задачу:

$$
v_{3 t}=B v_{3}-v_{1 s}-d_{\mathrm{Kp}} \varkappa_{1} v_{1 x x}+a_{k} \sin a_{k} \gamma_{\mathrm{\kappa p}}\left[\varkappa_{2} v_{h_{1}}+\frac{1}{6} v_{h_{1}}^{3}\right]=B v_{3}+F(t, x, s) .
$$

Условия разрешимости в классе периодических функций в данном случае будут иметь вид

$$
\int_{0}^{2 \pi / \sigma_{m}} \int_{0}^{2 \pi} F(t, x, s) \exp \left( \pm i m x \pm i \sigma_{m} t\right) d x d t=\int_{0}^{2 \pi / \sigma_{m}} \int_{0}^{2 \pi} F(t, x, s) \exp ( \pm i(m+1) x) d x d t=0 .
$$

Применяя это условие к (21), получаем систему, являющуюся нормальной формой:

$$
\left\{\begin{aligned}
z_{1}^{\prime}= & z_{1}\left(a_{k} \sin a_{k} \gamma_{\mathrm{\kappa p}} \varkappa_{2} \exp (i m h)+d_{\mathrm{\kappa p}} \varkappa_{1} m^{2}\right)+\frac{1}{2} \sin a_{k} \gamma_{\mathrm{\kappa p}} \exp (i m h)\left[\left|z_{1}\right|^{2}+2\left|z_{2}\right|^{2}\right] z_{1}, \\
z_{2}^{\prime}= & z_{2}\left(a_{k} \sin a_{k} \gamma_{\mathrm{\kappa p}} \varkappa_{2} \exp (i(m+1) h)+d_{\mathrm{\kappa p}} \varkappa_{1}(m+1)^{2}\right)+ \\
& +\frac{1}{2} \sin a_{k} \gamma_{\mathrm{kp}} \exp (i(m+1) h)\left[\left|z_{2}\right|^{2}+2\left|z_{1}\right|^{2}\right] z_{2} .
\end{aligned}\right.
$$

Преобразуем полученную нормальную форму, положив $z_{1}(s)=\rho_{1}(s) \exp \left(i \varphi_{1}(s)\right), z_{2}(s)=$ $\rho_{2}(s) \exp \left(i \varphi_{2}(s)\right)$. В результате нормальная форма принимает следующий вид

$$
\begin{array}{rlrl}
\rho_{1}^{\prime} & =g_{1} \rho_{1}+l_{1} \rho_{1}\left[\rho_{1}^{2}+2 \rho_{2}^{2}\right], & \rho_{2}^{\prime}=g_{2} \rho_{2}+l_{2} \rho_{2}\left[2 \rho_{1}^{2}+\rho_{2}^{2}\right], \\
\varphi_{1}^{\prime}=q_{1}+k_{1}\left[\rho_{1}^{2}+2 \rho_{2}^{2}\right], & \varphi_{2}^{\prime}=q_{2}+k_{2}\left[2 \rho_{1}^{2}+\rho_{2}^{2}\right],
\end{array}
$$


где

$$
\begin{gathered}
g_{1}=\nu \varkappa_{2} \cos m h+d_{\mathrm{Kp}} \varkappa_{1} m^{2}, \quad g_{2}=\nu \varkappa_{2} \cos (m+1) h+d_{\mathrm{Kp}} \varkappa_{1}(m+1)^{2}, \\
\nu=a_{k} \sin a_{k} \gamma_{\mathrm{Kp}}, \quad l_{1}=\frac{1}{2} \nu \cos m h, \quad l_{2}=\frac{1}{2} \nu \cos (m+1) h, \\
q_{1}=\nu \varkappa_{2} \sin m h, \quad q_{2}=\nu \varkappa_{2} \sin (m+1) h, \quad k_{1}=\frac{1}{2} \nu \sin m h, \quad k_{2}=\frac{1}{2} \nu \sin (m+1) h .
\end{gathered}
$$

Уточним вид коэффициентов нормальной формы при $h=\pi / 3$ и $h=\pi / 4$. Приведем их вид для $h=\pi / 3$ :

$$
\begin{gathered}
\nu=10, \quad g_{1}=-5 \varkappa_{2}+4 \varkappa_{1}, \quad g_{2}=-10 \varkappa_{2}+9 \varkappa_{1}, \quad l_{1}=-5 / 2, \quad l_{2}=-5, \\
q_{1}=5 \sqrt{3} \varkappa_{2}, \quad q_{2}=0, \quad k_{1}=5 \sqrt{3} / 2, \quad k_{2}=0 .
\end{gathered}
$$

В свою очередь, при $h=\pi / 4$ получим:

$$
\begin{gathered}
g_{1}=-\frac{7}{94}(16+9 \sqrt{2}) \varkappa_{2}+\frac{1}{94}(18+63 \sqrt{2}) \varkappa_{1}, \quad g_{2}=-\frac{7}{47}(9+8 \sqrt{2}) \varkappa_{2}+\frac{8}{47}(2+7 \sqrt{2}) \varkappa_{1}, \\
\nu=\frac{7}{47}(9+8 \sqrt{2}), \quad l_{1}=-\frac{7}{188}(16+9 \sqrt{2}), \quad l_{2}=-\frac{7}{94}(9+8 \sqrt{2}), \quad q_{1}=\frac{7}{94}(16+9 \sqrt{2}) \varkappa_{2}, \\
q_{2}=0, \quad k_{1}=\frac{7}{188}(16+9 \sqrt{2}), \quad k_{2}=0 .
\end{gathered}
$$

Особую роль при анализе полученной системы дифференциальных уравнений $(22),(23)$ играет замкнутая система для амплитуд (22). Она может иметь следующие нетривиальные состояния равновесия:

которое существует, если $g_{2} l_{2}<0$;

$$
S_{1}: \quad \rho_{1}=0, \quad \rho_{2}=\sqrt{-\frac{g_{2}}{l_{2}}}
$$

$$
S_{2}: \quad \rho_{1}=\sqrt{-\frac{g_{1}}{l_{1}}}, \quad \rho_{2}=0
$$

существующее при $g_{1} l_{1}<0$;

$$
S_{3}: \quad \rho_{1}=\sqrt{\frac{g_{1} l_{2}-2 l_{1} g_{2}}{3 l_{1} l_{2}}}, \quad \rho_{2}=\sqrt{\frac{l_{1} g_{2}-2 g_{1} l_{2}}{3 l_{1} l_{2}}},
$$

которое существует, если подкоренные выражения положительны.

Учитывая, что в рассматриваемых случаях $l_{1}, l_{2}<0$ при возможных значениях параметров, можно отметить, что состояние равновесия $S_{1}$ существует, если $g_{2}>0$, а $S_{2}$ существует при $g_{1}>0$. Добавим, что состояние равновесия $S_{3}$ существует, если $a=g_{2} l_{1}-2 g_{1} l_{2}>0$ и $b=g_{1} l_{2}-2 g_{2} l_{1}>0$.

Пусть $\rho_{1}=\eta_{1}, \rho_{2}=\eta_{2}$ - координаты одного из состояний равновесия. Тогда вопрос об его устойчивости, как хорошо известно, определяется расположением собственных значений матрицы Якоби, вычисленной в точке $\rho_{1}=\eta_{1}, \rho_{2}=\eta_{2}$.

В случае состояния равновесия $S_{3}$ матрица Якоби имеет следующий вид:

$$
J_{3}=\left(\begin{array}{cc}
\frac{2 b}{3 l_{2}} & \frac{4 \sqrt{a b}}{3 l_{2}} \\
\frac{4 \sqrt{a b}}{3 l_{1}} & \frac{2 a}{3 l_{1}}
\end{array}\right) ;
$$

при этом ее след и определитель отрицательны в силу положительности $a, b$ и отрицательности $l_{1}$ и $l_{2}$ :

$$
\operatorname{tr} J_{3}=\frac{2 b}{3 l_{2}}+\frac{2 a}{3 l_{1}}<0, \quad \operatorname{det} J_{3}=-\frac{4}{3} \cdot \frac{a b}{l_{1} l_{2}} .
$$

Напомним, что собственные значения матрицы $J$ находятся из уравнения

$$
\lambda^{2}-\operatorname{tr} J_{3} \lambda+\operatorname{det} J_{3}=0 .
$$

Следовательно, согласно критерию Рауса-Гурвица состояние равновесия $S_{3}$ всегда неустойчиво. 
Рассмотрим состояние равновесия $S_{1}$. В этом случае соответствующая матрица $J_{1}$ имеет вид

$$
J_{1}=\left(\begin{array}{cc}
g_{1}-2 \frac{l_{1} g_{2}}{l_{2}} & 0 \\
0 & -2 g_{2}
\end{array}\right) \text {. }
$$

Состояние равновесия $S_{1}$ является асимптотически устойчивым, если выполнено условие $g_{1}-$ $2 l_{1} g_{2} / l_{2}<0$. Рассуждая аналогично, несложно показать, что состояние равновесия $S_{2}$ асимптотически устойчиво, если $g_{2}-2 l_{2} g_{1} / l_{1}<0$.

Отметим, что из существования состояния равновесия $S_{3}$ следует асимптотическая устойчивость состояний равновесия $S_{1}$ и $S_{2}$. При отсутствии $S_{3}$ и наличии $S_{1}$ и $S_{2}$ одно из них асимптотически устойчиво, а второе неустойчиво.

Справедливо следующее утверждение.

\section{Теорема 7.}

1. Состояние равновесия $S_{1}$ существует, если $g_{2}>0$. Оно асимптотически устойчиво, если выполняется неравенство $g_{1}-2 l_{1} g_{2} / l_{2}<0$, и неустойчиво, когда $g_{1}-2 l_{1} g_{2} / l_{2}>0$.

2. Состояние равновесия $S_{2}$ существует, если $g_{1}>0$. Оно является асимптотически устойчивым, если $g_{2}-2 l_{2} g_{1} / l_{1}<0$, и неустойчивым в противном случае.

3. Для существования состояния равновесия $S_{3}$ необходимо, чтобы выполнялись условия $g_{1} l_{2}-2 l_{1} g_{2}>0$ и $l_{1} g_{2}-2 g_{1} l_{2}>0$. Если это состояние равновесия существует, то оно неустойчиво.

Конкретизируем этот результат применительно к нашему выбору параметров задачи.

Следствие 2. Пусть $h=\pi / 3$. Система дифференциальных уравнений (22) может иметь три нетривиальных состояния равновесия.

(1) Состояние равновесия

$$
S_{1}: \quad \rho_{1}=0, \quad \rho_{2}=\sqrt{\frac{9 \varkappa_{1}-10 \varkappa_{2}}{5}}
$$

существует, если $9 \varkappa_{1}-10 \varkappa_{2}>0$, и является асимптотически устойчивым, когда $\varkappa_{2}<\varkappa_{1}$.

(2) Состояние равновесия

$$
S_{2}: \quad \rho_{1}=\sqrt{\frac{8 \varkappa_{1}-10 \varkappa_{2}}{5}}, \quad \rho_{2}=0
$$

существует, если $4 \varkappa_{1}-5 \varkappa_{2}>0$, и является асимптотически устойчивым при выполнении условия $10 \varkappa_{2}-7 \varkappa_{1}<0$.

(3) Если выполнены условия $\varkappa_{1}>\varkappa_{2}$ и $7 \varkappa_{1}-10 \varkappa_{2}>0$, то существует неустойчивое состояние равновесия

$$
S_{3}: \quad \rho_{1}=\sqrt{\frac{5 \varkappa_{1}-5 \varkappa_{2}}{3}}, \quad \rho_{2}=\sqrt{\frac{7 \varkappa_{1}-10 \varkappa_{2}}{6}} .
$$

Следствие 3. Пусть $h=\pi / 4$.

(1) Состояние равновесия

$$
S_{1}: \quad \rho_{1}=0, \quad \rho_{2}=\sqrt{\frac{16-8 \sqrt{2}}{7} \varkappa_{1}-\varkappa_{2}}
$$

существует, если $(16-8 \sqrt{2}) \varkappa_{1}-7 \varkappa_{2}>0 ;$ при выполнении условия $7(288+209 \sqrt{2}) \varkappa_{2}<$ $(1358+1369 \sqrt{2}) \varkappa_{1}$ оно является асимптотически устойчивым.

(2) Состояние равновесия

$$
S_{2}: \quad \rho_{1}=\sqrt{\frac{18(\sqrt{2}-1)}{7} \varkappa_{1}-2 \varkappa_{2},}, \quad \rho_{2}=0
$$

существует при 18( $\sqrt{2}-1) \varkappa_{1}-14 \varkappa_{2}>0$ и является асимптотически устойчивым, если выполнено условие $7(288+209 \sqrt{2}) \varkappa_{2}<2(538+191 \sqrt{2}) \varkappa_{1}$. 
(3) Неустойчивое состояние равновесия $S_{3}$

$$
\begin{aligned}
& \rho_{1}=\sqrt{\frac{(2716+2738 \sqrt{2}) \varkappa_{1}-14(288+209 \sqrt{2}) \varkappa_{2}}{6048+4389 \sqrt{2}},} \\
& \rho_{2}=\sqrt{\frac{4(538+191 \sqrt{2}) \varkappa_{1}-14(288+209 \sqrt{2}) \varkappa_{2}}{6048+4389 \sqrt{2}}}
\end{aligned}
$$

существует, когда асимптотически устойчивы состояния равновесия $S_{1}$ и $S_{2}$.

Каждому состоянию равновесия $S_{1}, S_{2}, S_{3}$ с координатами $\rho_{1}=\eta_{1}, \rho_{2}=\eta_{2}$ соответствует решение системы (22), (23):

$$
\begin{array}{lll}
\rho_{1}=\eta_{1}, & \varphi_{1}=B\left(q_{1}+k_{1}\left[2 \rho_{2}^{2}+\rho_{1}^{2}\right]\right) s+\varphi_{1}, & \varphi_{1} \in \mathbb{R}, \\
\rho_{2}=\eta_{2}, & \varphi_{2}=B\left(q_{2}+k_{2}\left[2 \rho_{1}^{2}+\rho_{2}^{2}\right]\right) s+\varphi_{2}, & \varphi_{2} \in \mathbb{R} .
\end{array}
$$

Из приведенных теорем и результатов работ $[4,6]$ вытекает справедливость следующего утверждения.

Теорема 8. Пусть выполнены условия существования состояния равновесия $\left(S_{1}, S_{2}\right.$ или $\left.S_{3}\right)$. Тогда существует такое $\varepsilon_{0}>0$, что при всех $\varepsilon \in\left(0, \varepsilon_{0}\right)$ краевая задача $(17)$, (18) имеет решения c асимптотикой

$$
v(t, x, \varepsilon)=\varepsilon^{1 / 2} \eta_{1}\left(p_{1}+\overline{p_{1}}\right)+\varepsilon^{1 / 2} \eta_{2}\left(p_{2}+\overline{p_{2}}\right)+o(\varepsilon),
$$

где

$$
\begin{gathered}
p_{1}=\exp \left(i\left(\sigma_{m}+\varepsilon\left(q_{1}+k_{1} \psi_{1}\right)\right) t+i m x+i \varphi_{1}\right), \quad \psi_{1}=2 \eta_{2}^{2}+\eta_{1}^{2}, \quad \sigma_{m}=\operatorname{Im}\left(\lambda_{m, 1}\right), \\
p_{2}=\exp \left(i \varepsilon\left(q_{2}+k_{2} \psi_{2}\right) t+i(m+1) x+i \varphi_{2}\right), \quad \psi_{2}=2 \eta_{1}^{2}+\eta_{2}^{2}, \quad \varphi_{1}, \varphi_{2} \in \mathbb{R} .
\end{gathered}
$$

Эти решения наследуют устойчивость соответствующего состояния равновесия системзи (22).

6. Заключение. Среди рассмотренных состояний равновесия только состояния равновесия $a_{k}=\pi / 2+2 \pi k, k=0,1, \ldots$, приводят к содержательным бифуркационным задачам, когда из состояния равновесия могут бифурцировать пространственно неоднородные решения, т.е. решения, которые зависят существенным образом от $x$. Для этих однородных состояний равновесия могут реализовываться критические случаи трех типов $\left(\gamma=\gamma_{\text {кр }}\right)$. Первый из них таков: на мнимой оси находится одна простая пара чисто мнимых собственных значений. В этом случае бифурцирует периодическое по $t$ решение, которое также зависит от $x$. Для второго типа имеем критический случай двукратного нулевого собственного значения спектра устойчивости. Изучение соответствующей бифуркационной задачи показало возможность появления пространственно неоднородных состояний равновесия.

В работе изучена и бифуркационная краевая задача коразмерности 2 , когда на мнимой оси находятся пара чисто мнимых собственных значений и двукратное нулевое собственное значение. В этом случае получено двупараметрическое семейство периодических по $t$ решений, которые существенным образом зависят и от $x$.

\section{СПИСОК ЛИТЕРАТУРЫ}

1. Гукенхеймер Дж., Холмс Ф. Нелинейные колебания, динамические системы и бифуркации векторных полей. - М.-Ижевск: Ин-т компьют. исслед., 2002.

2. Кащенко С. А. Асимптотика пространственно неоднородных структур в когерентных оптических системах// Ж. вычисл. мат. мат. физ. - 1991. - 31, № 3. - С. 467-473.

3. Кащенко И. С., Кащенко С. А. Быстро осциллирующие пространственно неоднородные структуры в когерентных нелинейно-оптических системах// Докл. РАН. - 2010. - 435, № 1. - С. 14-17.

4. Колесов А. Ю., Куликов А. Н. Инвариантные торы нелинейных эволюционных уравнений. - Ярославль: ЯрГУ, 2005. 
5. Колесов А. Ю., Куликов А. Н., Розов Н. Х. Инвариантные торы одного класса точечных отображений: сохранение инвариантного тора при возмущениях// Диффер. уравн. - 2003. - 39, № 6. - С. $738-753$.

6. Колесов А. Ю., Розов Н. Х. Инвариантные торы нелинейных волновых уравнений. - М.: Физматлит, 2004.

7. Крейн С. Г. Линейные дифференциальные уравнения в банаховом пространстве. - М.: Наука, 1967.

8. Куликов A. Н. О гладких инвариантных многообразиях полугруппы нелинейных операторов в банаховом пространстве// в кн.: Исследования по устойчивости и теории колебаний. - Ярославль: ЯрГУ, 1976. - C. 114-129.

9. Марсден Джс, Мак-Кракен М. Бифуркация рождения цикла и ее приложения. - М.: Мир, 1980.

10. Мищенко Е. Ф., Садовничий В. А., Колесов А. Ю., Розов Н. Х. Автоволновые процессы в нелинейных средах с диффузией. - М.: Физматлит, 2005.

11. Разгулин А. В. Об автоколебаниях в нелинейной параболической задаче с преобразованным аргументом// Ж. вычисл. мат. мат. физ. - 1993. - 33, № 1. - С. 69-80.

12. Скубачевский A. Л. О бифуркации Хопфа для квазилинейного параболического функциональнодифференциального уравнения// Диффер. уравн. - 1998. - 34, № 10. - С. 1394-1401.

13. Соболевский П. Е. Об уравнениях параболического типа в банаховом пространстве// Тр. Моск. мат. об-ва. - 1967. - 10. - С. 297-350.

Ковалева Анастасия Михайловна

Ярославский государственный университет им. П. Г. Демидова

E-mail: anastasia2kovaleva@gmail.com 